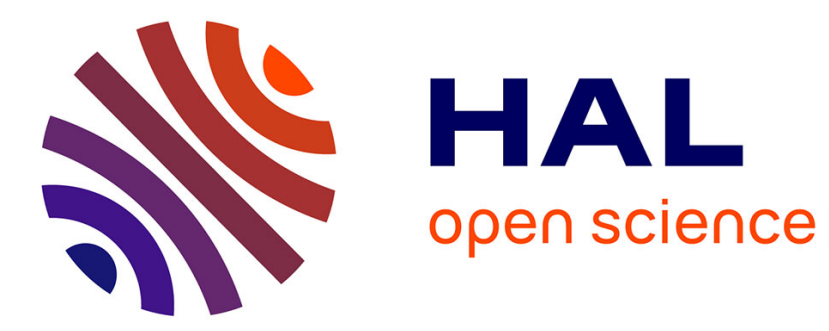

\title{
Absentéisme et conditions de travail au féminin : Une frontière méconnue au sein des organisations \\ Gregor Bouville
}

\section{To cite this version:}

Gregor Bouville. Absentéisme et conditions de travail au féminin : Une frontière méconnue au sein des organisations. RIMHE : Revue Interdisciplinaire Management, Homme(s) \& Entreprise, 2014, 11 (2), pp.3-22. 10.3917/rimhe.011.0003 . hal-03227030

\section{HAL Id: hal-03227030 https://hal.science/hal-03227030}

Submitted on 16 May 2021

HAL is a multi-disciplinary open access archive for the deposit and dissemination of scientific research documents, whether they are published or not. The documents may come from teaching and research institutions in France or abroad, or from public or private research centers.
L'archive ouverte pluridisciplinaire HAL, est destinée au dépôt et à la diffusion de documents scientifiques de niveau recherche, publiés ou non, émanant des établissements d'enseignement et de recherche français ou étrangers, des laboratoires publics ou privés. 


\section{Absentéisme et conditions de travail au féminin : une frontière méconnue au sein des organisations}

Gregor BOUVILLE ${ }^{1}$

\section{Résumé:}

Un phénomène distingue nettement les hommes et les femmes dans les lieux de travail : l'absentéisme. La littérature établit sans conteste que les femmes s'absentent du travail plus que les hommes. Pour expliquer la différence entre taux féminin et masculin d'absentéisme au travail, certains chercheurs mettent en exergue des déterminants hors travail tels que les responsabilités familiales. En fait, la relation entre les responsabilités familiales et l'absentéisme n'est pas clairement établie. D'autres chercheurs par contre mettent en évidence des facteurs liés au fonctionnement du lieu de travail : organisation du travail, conditions de travail, relations de travail, ressources de l'emploi. Nous examinons ces facteurs et montrons qu'il existe une frontière méconnue en France au sein du monde du travail en fonction du sexe du salarié. L'organisation du travail, les conditions de travail, les relations sociales et les ressources de l'emploi sont différentes selon le sexe du salarié et sont les principaux déterminants de l'absentéisme féminin.

Mots-clés: sexe, division sexuelle du travail, organisation du travail, conditions de travail, absentéisme

Pour citer cet article: Bouville, G. (2014), Absentéisme et conditions de travail au féminin : une frontière méconnue au sein des organisations, Revue Interdisciplinaire Management, Homme $(s)$ \& Entreprise, Vol. 11, 2014, pp. 3-22.

\footnotetext{
${ }^{1}$ Maître de conférences en sciences de gestion Université Paris-Dauphine DRM Management \& Organisation-UMR CNRS 7088 gregor.bouville@dauphine.fr Tel : 0144054911
} 


\title{
Women absenteeism and working conditions: an unknown boundary within the workplace
}

\begin{abstract}
:
A phenomenon clearly distinguishes men from women as regards work places: absenteeism. The current literature establishes as an unquestionable fact that women are more absent from work then men. To explain the discrepancies between men and women absenteeism rate, some researchers highlight off-work determinants such as family responsibilities (duties). Actually, the connexion between family responsibilities and absenteeism is not clearly established. On the other hand, other researchers point up factors related to the functioning of the work place: work organization, working conditions, work relations, job resources. We examine these factors and show that, within the working world, there exists an unheard-of boundary in France that depends on employee's sex. Work organization, working conditions, social relations and job resources are different depending on the employee's sex and are the main determinants of women absenteeism.
\end{abstract}

Key words: sex, sexual division of work, work organization, working conditions, absenteeism 
Un phénomène distingue nettement les hommes et les femmes dans les lieux de travail: l'absentéisme. L'absentéisme ${ }^{2}$ touche davantage les femmes que les hommes (Vlassenko et Willard, 1984; Farell et Stamm, 1988; Côté et Haccoun, 1991). Toutefois, Deguire et Messing (1995) dénoncent le biais sexiste de ces études. En effet, le sexe y est traité comme une variable explicative en soi, sans que ne soient considérées les conditions de travail et de vie et les spécificités biologiques qui sont associées au sexe.

On peut distinguer deux types d'approche de ce phénomène : une approche non basée sur le travail et l'autre au contraire axée sur le travail. Les chercheurs optant pour une approche non axée sur le travail soutiennent que la différence dans les comportements d'absence masculin et féminin est due principalement aux difficultés qu'ont les femmes à trouver un équilibre entre travail et responsabilités familiales et domestiques (Bratberg et alii, 2002; Vistnes, 1997). Cette différence reflèterait également un certain nombre de caractéristiques de santé et personnelles différenciant les hommes et les femmes (Mastekaasa et Olsen, 1998).

D'un autre côté, les chercheurs optant pour une approche axée sur le travail considèrent que le différentiel entre les taux d'absentéisme masculin et féminin est le résultat de déterminants inhérents au lieu de travail (Messing et alii, 1998; Laksonenen et alii, 2008; Hensing et Alexanderson, 2004). Les femmes sont plus absentes que les hommes à cause «d'emplois moins intéressants et de conditions de travail plus mauvaises »(Patton et Johns, 2007, p. 1581).

Nous examinons dans un premier temps les argumentations développées et les vérifications opérées par les tenants de ces deux approches. Nous exposons dans un deuxième temps nos hypothèses et notre méthodologie. Dans un troisième temps, nous présentons nos résultats. Nous les discutons, dans un dernier paragraphe.

\section{Les causes de l'absentéisme féminin: déterminants extérieurs au travail ou inhérents au travail?}

Deguire et Messing (1995) estiment que les spécificités biologiques des femmes ne sont pas prises en compte. Ichino et Moretti (2009) montrent ainsi que la probabilité de s'absenter pour les femmes est fonction du cycle menstruel. Ce résultat n'est plus constaté pour les femmes de plus de 45 ans. Ichino et Moretti (2009) en concluent que les cycles menstruels expliquent une part significative (environ deux tiers pour la fréquence des absences et un tiers pour la durée des absences) de la différence entre le taux d'absentéisme des hommes et des femmes.

D'autres chercheurs expliquent la différence de taux d'absentéisme des hommes et des femmes par la difficulté à concilier pour les femmes leur vie professionnelle et leur vie privée (Goff et alii, 1990). Cette caractéristique, souvent présentée comme une spécificité féminine, est générée par une inégale répartition des tâches domestiques et familiales entre les hommes et les femmes. Rappelons qu'en

\footnotetext{
${ }^{2}$ L'absentéisme est mesuré en ne tenant pas compte des congés-maternités.
} 
France, les tâches domestiques et familiales sont très inégalement partagées en défaveur des femmes (Alis et Dumas, 2003 ; Dumas, 2008 ; Brugueilles et Sebille, 2009). L'étude de la relation entre responsabilités familiales et absentéisme a donné lieu à plusieurs études (Erickson et alii, 2000 ; Goff et alii, 1990 ; Mastekaasa, 2000 ; Bradberg et alii, 2002). Dans l'étude de Goff et alii (1990), le lien entre responsabilités familiales et absentéisme est significatif, mais aucune variable de contrôle (âge, ancienneté, sexe) n'est incluse dans les analyses statistiques. Les résultats des travaux de Mastekaasa (2000) sont plus nuancés. Les femmes et les hommes avec enfants ont un taux d'absentéisme légèrement supérieur à celui des femmes et des hommes sans enfants. Le taux d'absentéisme décroît, cependant, au fur et à mesure que l'âge de l'enfant avance. Il devient même inférieur à celui des femmes et des hommes sans enfants après que l'âge de leur plus jeune enfant ait franchi le seuil des dix ans. Dans l'étude d'Erickson et alii (2000), la relation entre ces deux variables n'est plus significative après contrôle des variables «sexe», «âge » et «niveau d'éducation ». Enfin, Bradberg et alii (2002), en contrôlant l'effet des variables « sexe », «âge » et «niveau d'éducation », montrent que le taux d'absentéisme des femmes diminue lorsque le nombre de leurs enfants augmente. Ceci montre que la variable « responsabilités familiales » a tendance à saisir des différences plus générales entre individus liées à l'âge, au sexe ou au niveau d'éducation. Ce ne serait pas tant les responsabilités familiales qui auraient un effet sur l'absentéisme que leur inégale répartition entre les hommes et les femmes, elle-même dépendante de l'âge et du niveau d'éducation des conjoints.

Cet ensemble de recherches ne réussit pas à démontrer que la différence homme-femme dans le comportement d'absence-maladie est essentiellement causé par la difficulté des femmes à gérer simultanément engagement professionnel et responsabilités familiales (Masterkaasa et Olsen, 1998; Erickson et alii, 2000; Masterkaasa, 2000; Bratberg et alii, 2002). En fait, la relation entre les responsabilités familiales et l'absentéisme n'a pas été clairement établie. Par contre, l'explication de la différence entre taux féminin et masculin d'absentéisme au travail par des modes d'organisation du travail, de conditions de travail et de relations sociales spécifiques semble prometteuse et mérite d'être explorée (Laksonenen et alii, 2008)

Partant du constat que les femmes sont concentrées dans des secteurs professionnels se caractérisant par des conditions de travail difficiles - par exemple, dans le cas français, les femmes sont concentrées dans cinq secteurs socioprofessionnels : services de proximité, santé, services sociaux, enseignement, secteurs du commerce et de la grande distribution- ${ }^{3}$, quelques rares études ont cherché à montrer que l'effet a priori du sexe sur l'absentéisme était en fait un effet de confusion, reflet de la division sexuée du travail (Messing et alii, 1998 ; Messing et alii, 2011). D'autres chercheurs ont adopté une approche sexuée pour étudier les effets de déterminants liés au lieu de travail (facteurs psychosociaux et

\footnotetext{
${ }^{3}$ Source : Chappert F. (2009, p. 17-23), Genre et conditions de travail, Etudes et documents, ANACT.
} 
conditions de travail) sur l'absentéisme (par exemple, North et alii, 1996; Vaananen et alii, 2003; Niedhammer et alii, 2006; Melchior et alii, 2003; Messing et alii, 1998; Laksonenen et alii, 2008). Néanmoins, en ce qui concerne les facteurs psychosociaux (demande psychologique et soutien social $^{4}$ ), les résultats de ces études sont en partie contradictoires. Dans l'étude Whitehall II réalisée auprès de fonctionnaires londoniens, la demande psychologique n'entretient aucune relation sexuée avec les absences-maladie (North et alii, 1996). Inversement, Niedhammer et alii (2006), Melchior et alii (2003) ont démontré qu'une demande psychologique forte accrôtt les absences-maladies des femmes mais pas celles des hommes. Toutefois, dans l'étude de Laksonenen et alii (2008), la demande psychologique n'a pas d'effet sur la différence entre l'absentéisme auto-déclaré ou médicalement attesté des hommes et des femmes. Dans une étude centrée sur le soutien social, réalisée par Melchior et alii (2003), un soutien social faible a un impact sur les très longues absences (supérieures à 21 jours) des hommes mais pas sur celles des femmes. De plus, dans l'étude menée par North et alii (1996), un fort soutien social a un impact à la fois sur les courtes (inférieures à 7 jours) et les longues (supérieures à 7 jours) absences-maladie des hommes et des femmes. Dans l'étude conduite par Vaananen et alii (2003), un fort soutien des collègues a un impact sur les très longues absencesmaladies (supérieures à 22 jours) des hommes mais pas sur celles des femmes. Dans la même étude, le soutien du supérieur hiérarchique n'a aucun effet sur les très longues absences-maladies (supérieures à 22 jours) des hommes ou des femmes.

Quant au facteur «conditions de travail » (environnement physique de travail, pénibilité physique et mentale), Messing et alii (1998) ont montré que les différences en termes d'absences-maladies entre les hommes et les femmes étaient principalement liées à aux caractéristiques sexuées de l'environnement physique de travail. De la même façon, après introduction dans le modèle de régression de la charge physique, la différence entre l'absentéisme auto-déclaré ou médicalement attesté des hommes et des femmes diminue (Laksonenen et alii, 2008). Ainsi, d'après ce résultat, dès lors qu'est prise en compte, dans le modèle de régression, l'exposition différenciée à une charge physique élevée des hommes et des femmes, le sexe n'est plus un facteur explicatif important de la différence entre l'absentéisme auto-déclaré ou médicalement attesté des hommes et des femmes.

Enfin, concernant les ressources de l'emploi, à notre connaissance, aucune étude n'en a testé l'influence sexuée sur l'absentéisme. Par ressources de l'emploi, nous entendrons l'ensemble des moyens humains (environnement de travail dans lequel le salarié est entouré d'un nombre suffisant de collègues ou de collaborateurs), informationnels (environnement de travail dans lequel le salarié dispose d'informations claires et suffisantes) et cognitifs (environnement de travail dans lequel le

\footnotetext{
${ }^{4}$ Ces deux variables sont des dimensions du modèle de Karasek et Theorell (1990). La demande psychologique renvoie à la quantité de travail à accomplir (« on me demande de réaliser une quantité excessive de travail »), aux exigences mentales («mon travail nécessite de longues périodes de concentration intense ») et aux contraintes de temps liées à ce travail («je dispose du temps nécessaire pour exécuter mon travail »). Le soutien social (des collègues et du supérieur), incluant deux composantes : soutien instrumental et soutien socioémotionnel. Le manque de soutien social constitue un facteur de risque pour la santé.
} 
salarié dispose d'une formation suffisante et adaptée) fournis par l'organisation, susceptibles de réduire les contraintes de l'organisation ou de contribuer à l'épanouissement personnel ou de concourir à l'atteinte des objectifs fixés au salarié (Bouville, 2009). Nous pouvons supposer qu'une répartition sexuée des ressources de l'emploi induira un effet différencié sur l'absentéisme des hommes et des femmes.

Comme l'ont noté Patton et Johns (2007, p. 1583), «nous sommes confrontés à des études qui suggèrent que les femmes s'absentent plus que les hommes mais qui fournissent peu d'explications sur le pourquoi ». De plus, ces rares études sexuées sur les déterminants de l'absentéisme aboutissent à des résultats divergents. De nouvelles investigations sont donc nécessaires pour comprendre les différences dans les comportements d'absence-maladie des hommes et des femmes. En effet, si les facteurs hors travail jouent un rôle important dans la difficile conciliation entre vie privée et vie professionnelle, les facteurs inhérents au lieu de travail semblent fournir une piste de recherche prometteuse pour expliquer les différences entre hommes et femmes. Toutefois, contrairement aux études précédentes, qui se focalisent sur les seules conditions de travail ou sur les variables « demande psychologique » et «latitude décisionnelle $»^{5}$ en référence au modèle de Karasek et Theorell (1990), nous explorerons un spectre plus large de déterminants organisationnels de l'absentéisme intégrant non seulement les conditions de travail mais aussi l'organisation du travail, les relations sociales et les ressources de l'emploi. Par organisation du travail, nous entendons la manière dont le travail est divisé et coordonné dans l'entreprise (Barreau, 2005). Celle-ci inclut quatre composantes : le contenu du travail, la coordination du travail, l'intensité du travail et l'organisation du temps de travail (Bouville, 2009). Les conditions de travail se définissent comme l'ensemble des pénibilités (physiques et mentales), les nuisances et les risques professionnels de l'environnement physique de travail auxquels sont exposés les salariés dans le cadre de leur travail (Gollac et Volkoff, 2007). Les relations sociales caractérisent l'amplitude du contrôle hiérarchique et du soutien social au sein d'une organisation (soutien du supérieur et soutien des collègues). De manière à comparer nos résultats à ceux d'études précédentes portant sur des analyses sexuées des déterminants organisationnels de l'absentéisme, nous reprendrons la mesure de l'absentéisme présente dans ces études (Melchior et alii, 2003 ; North et alii, 1996 ; Vaananen et alii, 2003) : absences-maladie courtes, longues et très longues en fonction du nombre annuel de jours d'absence. Notre démonstration reposera sur plusieurs analyses quantitatives. Tout d'abord, nous chercherons à montrer, qu'à organisation, conditions de travail, relations sociales et ressources de l'emploi identiques entre hommes et femmes, c'est-à-dire toutes choses étant égales par ailleurs, l'effet de la variable «sexe »sur l'absentéisme est faible. Ensuite, nous chercherons à

\footnotetext{
${ }^{5}$ La latitude décisionnelle fait référence à la possibilité de choisir les modes opératoires, à la capacité de peser sur les décisions et à la possibilité d'utiliser et de développer ses compétences. Elle comprend deux composantes, l'une tenant à la possibilité de choisir les modes opératoires et à la capacité de peser sur les décisions ( $\mathrm{j}$ 'ai la liberté de décider comment faire mon travail »), l'autre se situant sur le plan de l'accomplissement de soi au travers de la possibilité d'utiliser et de développer ses compétences («je fais preuve de créativité dans mon travail »).
} 
observer des différences sexuées dans la nature et l'amplitude des effets des déterminants organisationnels sur l'absentéisme en menant des analyses séparément pour les hommes et pour les femmes.

\section{Méthodologie de la recherche}

Ce travail est basé sur l'enquête française Sumer 2003. Cette enquête a été réalisée de juin 2002 à fin 2003. Rappelons que la décennie 1993-2003 est marquée par des performances économiques et sociales de la France supérieures à la moyenne européenne ${ }^{6}$. Pendant cette période, le taux de chômage y a baissé de 1,9 point contre 1,2 point pour l'ensemble de l'Union Européenne tandis que la productivité horaire du travail et le PIB y ont progressé plus rapidement (respectivement, près de $1 \%$ contre $0,4 \%$ et $2,13 \%$ contre $2,08 \%)^{7}$. Cette période est aussi marquée par le passage aux 35 heures. Dans le cadre de cette enquête, 1792 médecins du travail, représentant plus de $20 \%$ des médecins du travail en France, ont choisi au hasard 56314 salariés dont 49984 ont répondu au questionnaire. Le champ d'application de l'enquête comprend l'ensemble des salariés français y compris ceux des hôpitaux publics, de l'ex-grande entreprise publique EDF-GDF (Electricité et Gaz de France), de La Poste, de la SNCF et d'Air France. Son champ d'application ne couvre pas, toutefois, les fonctions publiques territoriale et d'Etat, le secteur des transports, des mines, de la pêche et des télécommunications (France Télécom). L'enquête couvre 17,5 millions des 21,7 millions de salariés en France, soit $80 \%$ des salariés français. L'enquête Sumer est divisée en deux parties. La première partie de l'enquête dresse un état des lieux des principales contraintes organisationnelles des salariés et de leur environnement physique. Les données sont recueillies par le médecin du travail lors de l'entretien médico-professionnel au cours des visites périodiques, et font également appel à la connaissance des postes de travail qu'il a acquise au cours de son tiers-temps. La deuxième partie de l'enquête repose sur un auto-questionnaire, proposé à un salarié sur deux, qui lui permet d'exprimer son avis sur sa situation de travail. Cet auto-questionnaire inclut le Job Content Questionnaire ou JCQ de Karasek et Theorell (1990), des questions sur les rythmes du temps de travail, sur la satisfaction et la santé au travail. Cette méthode consiste donc à retenir deux sources d'information indépendantes, pour la santé d'une part, les conditions de travail, d'autre part. Elle vise à réduire le risque de biais systématique lié à l'endogénéité de la description des conditions de travail par le salarié (Coutrot et Wolff, 2005). Les déterminants organisationnels de l'absentéisme caractéristiques de l'organisation du travail, des conditions de travail, des relations sociales et des ressources de l'emploi sont présentées dans le tableau 1.

\footnotetext{
${ }^{6}$ Source : IRES (2005), Les mutations de l'emploi en France, Paris, La Découverte.

${ }^{7}$ Source : Idem
} 
Tableau 1: Les variables organisationnelles étudiées

\begin{tabular}{|l|l|}
\hline $\begin{array}{l}\text { Organisation du travail } \\
\text { Temps de travail, demande psychologique, } \\
\text { autonomie décisionnelle, utilisation et } \\
\text { développement des compétences, horaires } \\
\text { individualisés }\end{array}$ & $\begin{array}{l}\text { Conditions de travail } \\
\text { Postures douloureuses, nuisances thermiques, } \\
\text { nuisance sonores, agressions de la part du } \\
\text { public, tensions avec le public, harcèlement } \\
\text { moral }\end{array}$ \\
\hline $\begin{array}{l}\text { Ressources de l'emploi } \\
\text { moyens humains, moyens informationnels, } \\
\text { moyens cognitifs }\end{array}$ & $\begin{array}{l}\text { Relations sociales } \\
\text { soutien des collègues, soutien du supérieur, } \\
\text { contrôle hiérarchique }\end{array}$ \\
\hline
\end{tabular}

\subsection{Mesures des variables}

- Mesure de l'absentéisme :

Afin de mesurer l'absentéisme, nous utiliserons une variable nominale composée de quatre catégories: 0 jour d'absence, 1-7 jours (absences courtes d'une durée cumulée comprise entre 1 et 7 jours), 8-21 jours (absences longues d'une durée cumulée comprise entre 8 et 21 jours), > 21 jours (absences très longues d'une durée cumulée supérieure à 21 jours). Cette catégorisation de l'absentéisme se justifie par le fait que les déterminants de l'absentéisme diffèrent selon la durée des absences (Marmot et alii, 1995; North et alii, 1996; Melchior et alii, 2003). Cette catégorisation est similaire à celles utilisées dans plusieurs études en épidémiologie (l'étude Gazel, Melchior et alii, 2003; l'étude Whitehall II, North et alii, 1996 et une étude finnoise, Vaananen et alii, 2003). Cette similarité nous permet de comparer nos résultats à ceux des études précédentes. La variable à expliquer (l'absentéisme maladie) repose sur l'auto-déclaration des salariés, ce qui pourrait introduire certains biais liés à la sousdéclaration des salariés. Cependant, les recherches de Ferrie et alii (2005) et de Voss et alii (2008) ont montré que le recensement des absences par l'auto-déclaration pouvait constituer une approximation relativement bonne du recensement obtenu par l'intermédiaire d'une base de données.

- Mesures des variables liées à l'organisation du travail :

Le temps de travail correspond à la durée hebdomadaire moyenne au travail.

La demande psychologique a été mesurée par les neufs items du Job Content Questionnaire (JCQ) de Karasek et Theorell (1990). Les participants répondaient sur une échelle à 4 points. La cohérence interne est correcte $(\alpha=0,78)$.

L'autonomie décisionnelle a été mesurée par les trois items du Job Content Questionnaire (JCQ) de Karasek et Theorell (1990). Les participants répondaient sur une échelle à 4 points. La cohérence interne est acceptable $(\alpha=0,60)$. Cette valeur relativement faible de l'alpha Cronbach pour la mesure de l'autonomie décisionnelle est convergente avec celle obtenue dans une étude portante sur 2064 salariés hollandais et dans laquelle la cohérence interne est égale à 0,65 (de Jonge et alii, 2000). 
L'utilisation et le développement des compétences a été mesurée par les six items du Job Content Questionnaire (JCQ) de Karasek et Theorell (1990). Les participants répondaient sur une échelle à 4 points. La cohérence interne est correcte $(\alpha=0,70)$.

Les horaires individualisés sont mesurés par l'item suivant: «Pouvez-vous choisir vos horaires de travail ? ». Les participants répondaient sur une échelle à 3 points.

- Mesures des variables liées aux conditions de travail :

Les postures douloureuses est une variable dichotomique mesurée par l'item suivant : «Avezvous été exposé à une posture douloureuse suivante : déplacement à pied dans le travail, mouvements répétitifs, position debout, position à genoux, maintien de bras en l'air, au moins 20 heures lors de la semaine précédente ? ».

Les nuisances thermiques est une variable dichotomique mesurée par l'item suivant : «Avezvous été exposé à une nuisance thermique suivante : travail à une température $>24^{\circ} \mathrm{C},<-15$ ${ }^{\circ} \mathrm{C}$, travail en milieu humide ou travail à l'extérieur, au moins 20 heures lors de la semaine précédente ?»

Les nuisances sonores est une variable dichotomique mesurée par l'item suivant : «Avezvous été exposé au moins à une pollution sonore $: \geq 85$ décibels, des chocs, des impulsions, autre bruit gênant, au moins 20 heures au sein de la semaine précédente ?».

L'agression du public est une variable dichotomique mesurée par l'item suivant : «Dans les douze derniers mois, avez-vous été exposé à une agression verbale, physique ou sexuelle de la part du public ?». Les réponses possibles étaient « Oui » ou « Non ».

Les tensions avec le public a été mesurée par l'item suivant : «Vivez-vous des situations de tension dans vos rapports avec le public ? ». Les participants répondaient sur une échelle à 4 points.

Le harcèlement moral est une variable dichotomique mesurée par l'item suivant : «Avez-vous été exposé à une forme de situations difficiles suivantes (parmi les 10 formes de harcèlement proposées par Leymann (1996)) ?».

- Mesures des variables liées aux relations sociales :

Le soutien des collègues et celui du supérieur ont été mesurés par les huit items du Job Content Questionnaire (JCQ) de Karasek et Theorell (1990). La cohérence interne des deux variables est élevée (respectivement $\alpha=0,83$ et $\alpha=0,78$ ).

Le contrôle hiérarchique est une variable dichotomique mesurée au travers de l'item suivant : «Contrôle ou surveillance permanents exercés par la hiérarchie ». Les réponses possibles étaient « Oui » ou « Non ». 
- Mesures des variables liées aux ressources de l'emploi :

Les ressources de l'emploi sont mesurées par les variables dichotomiques («Oui» ou «Non ») suivantes :

- Moyens humains : «Pour effectuer correctement votre travail, avez-vous en général un nombre de collègues ou de collaborateurs suffisants ?».

- Moyens informationnels : «Pour effectuer correctement votre travail, avez-vous en général des informations claires et suffisantes?».

- Moyens cognitifs : «Pour effectuer correctement votre travail, avez-vous en général une formation suffisante et adaptée? ».

\subsection{Analyse statistique}

Les analyses statistiques ont été effectuées sur la base de régressions logistiques multinomiales pour plusieurs raisons. Tout d'abord, les données d'absence ne sont pas normalement distribuées (Baba, 1990). De plus, les techniques de normalisation des données d'absence, tels que la transformation par la racine carré ou le logarithme, ne sont pas opérantes (Watson et alii, 1985). Par ailleurs, les déterminants organisationnels et l'intensité de leurs effets varient en fonction de la durée des absences (Marmot et alii, 1995; North et alii, 1996; Melchior et alii, 2003). Ce dernier constat nous a incité à opter non pas pour la régression logistique ordinale mais pour la régression logistique multinomiale, de manière à identifier les déterminants de l'absentéisme en fonction des différentes modalités de la variable «absentéisme». Nous n'avons pas non plus mobilisé la technique de la régression log-linéaire de Poisson comme le font certaines recherches épidémiologiques (Marmot et alii, 1995; North et alii, 1996; Melchior et alii, 2003) pour deux raisons. Premièrement, la régression log-linéaire de Poisson requiert des variables indépendantes nominales. Ainsi, si nous catégorisons des variables continues en variables nominales, afin qu'elles s'adaptent à notre modèle de régression log-linéaire de Poisson, de l'information peut être perdue et les corrélations risquent d'être atténuées, ce qui pourrait in fine fausser nos résultats. Deuxièmement, le recours à la régression log-linéaire de Poisson oblige à effectuer trois régressions (pour chacune des catégories d'absence), au lieu d'une en utilisant la technique de la régression multinomiale, ce qui conduirait de nouveau à perdre de l'information. Par conséquent, l'analyse multivariée a été réalisée via une régression logistique multinomiale en contrôlant les facteurs de confusion potentiels (taille de l'établissement, secteur d'activité, sexe, âge, ancienneté, catégorie socioprofessionnelle (CSP), statut de l'emploi, type de temps de travail) sur deux échantillons ( $n=14241$ hommes et $n=10245$ femmes). 


\section{Résultats}

Le tableau 2 expose la répartition des salariés en fonction de leurs caractéristiques individuelles (ancienneté, catégorie d'âge, sexe) et sociétales (CSP, fonction exercée, statut de l'emploi et type de temps de travail) pour les hommes et pour les femmes.

Tableau 2 : Pourcentage de salariés selon leurs caractéristiques individuelles et sociétales (en pourcentage)

\section{Catégorie socioprofessionnelle}

(CSP)

Femmes $(n=10245)$

Cadres, professions Supérieures

Professions intermédiaires

17

27

10

46

Ouvriers

Fonction exercée

Production, fabrication, chantier

Installation, entretien, réparation

Nettoyage, gardiennage, travail

ménager

Manutention, magasinage,

transports

Guichet, saisie, standard,

secrétariat

Gestion, comptabilité,

administration

Commerce, vente, technico-

commercial

Recherche, étude, méthode,

informatique

Direction générale

Enseignement, santé, information et autre cas

\section{Ancienneté}

Moins d'un an

Entre 1 et 3 ans

Entre 4 et 10 ans

Plus de 10 ans

Tranche d'âge du salarié

$<25$ ans

25-29 ans

30-39 ans

40-49 ans

$\geq 50$ ans

\section{Statut de l'emploi}

Apprenti

Stagiaire ou contrat aidé 


\begin{tabular}{lcc}
\hline Intérimaire & 3 & 2 \\
Contrat à durée déterminée (CDD) & 2 & 4 \\
Contrat à durée indéterminée & 90 & 80 \\
(CDI) & 3 & 12 \\
Fonctionnaire & & \\
Type de contrat de travail & 96 & 74 \\
Temps complet & 4 & 26 \\
Temps partiel & & \\
Secteur d'activité & 2 & 1 \\
Agriculture & 40 & 20 \\
Industrie & 8 & 1 \\
Construction & 50 & 78 \\
Tertiaire & & 21 \\
Taille de l'établissement & 16 & 23 \\
$1-9$ salariés & 23 & 17 \\
$10-49$ salariés & 18 & 11 \\
$50-199$ salariés & 12 & 27 \\
$200-499$ salariés & 30 & \\
$\geq 500$ salariés & & \\
\hline
\end{tabular}

Tableau 3 : Sexe des salariés et absentéisme (en pourcentage)

\begin{tabular}{lccc}
\hline & $\mathbf{1 - 7} \mathbf{j}^{\mathbf{1}}$ & $\mathbf{8 - 2 1 \mathbf { j } ^ { \mathbf { 1 } }}$ & $\mathbf{2 1 \mathbf { j }}^{\mathbf{1}}$ \\
\cline { 2 - 4 } Sexe & & & \\
Homme & 40,6 & 26,5 & 13,3 \\
Femme & 43,7 & 28,3 & 16,0 \\
Ensemble & 42,0 & 27,3 & 14,5 \\
\hline
\end{tabular}

${ }^{1}:$ Test du Khi² significatif $(p<0,05)$

Le tableau 2 fournit plusieurs informations: les femmes sont sur-représentées par rapport aux hommes dans la catégorie socioprofessionnelle des employés et dans les professions liées à l'enseignement et à la santé (respectivement, $50 \%, 16 \%$ et $21 \%$ ). Inversement, les femmes sont sous-représentées dans la catégorie des ouvriers (respectivement, $14 \%$ et $16 \%$ ). Par ailleurs, l'absentéisme touche significativement davantage les femmes que les hommes, ce quelque soit la forme prise par l'absentéisme $\left(\mathrm{Khi}^{2}, p<.05\right.$, tableau 3).

Des analyses de régression logistique univariées et multivariées ont ensuite effectuées (tableaux 4, 5, 6, 7). Les pseudo- $\mathrm{R}^{2}$ de Nagelkerke sont relativement faibles (tableaux 4, 5, 6, 7). Ceci est un résultat 
normal et même attendu ${ }^{8}$ (Hosmer et Lemeshow, 2000; Mittblöck et Heinzl, 2001) dans le cas des modèles de régression logistique. Les mesures des pseudo- $\mathrm{R}^{2}$ de Mc Fadden, de Cox et Snell et de Nagelkerke adoptent des valeurs basses ou modérées bien que le modèle ajusté soit approprié (Mittblöck et Heinzl, 2001). Comme le note Pampel (2000, p. 50), «les chercheurs doivent utiliser ces mesures seulement comme des indicateurs frustres sans attribuer une grande importance à un seuil précis ».

Le tableau 4 fournit les résultats de la régression logistique multinomiale testant l'association univariée entre la variable «sexe» et l'absentéisme, toutes choses étant égales par ailleurs ${ }^{9}$ pour l'ensemble de l'échantillon. La variable «sexe» est fortement associée avec les absences courtes, longues, et très longues.

Le tableau 5 fournit les résultats de la régression logistique multinomiale testant l'association multivariée entre la variable sexe et l'absentéisme, toutes choses étant égales par ailleurs ${ }^{10}$ pour l'ensemble de l'échantillon. La variable «sexe » est fortement associée avec les absences courtes et longues, plus faiblement avec les absences très longues.

Les analyses multivariées conduites séparément pour les hommes et les femmes montrent que les variables liées aux conditions de travail et aux ressources de l'emploi ont un effet très important sur l'absentéisme. Les agressions du public, le harcèlement moral au travail et les nuisances thermiques ont un effet significatif important sur l'absentéisme des femmes et des hommes. Une analyse plus fine montre que, comparativement aux femmes, pour lesquelles le harcèlement au travail a un effet significatif sur les trois catégories d'absence (respectivement, $\beta=.151, p<.1$ pour les absences de 1$7 \mathrm{j} ; \beta=.250, p<.1$ pour les absences de $8-21 \mathrm{j} ; \beta=.356, p<.01$ pour les absences de plus de $22 \mathrm{j}$ ), pour les hommes, le harcèlement au travail est associé avec la seule catégorie des absences très longues $(\beta=.308, p<.05$ pour les absences de plus de $22 \mathrm{j})$. Par ailleurs, les nuisances sonores sont respectivement associées avec la catégorie des absences longues pour les hommes $(\beta=.240, p<.1$ pour les absences de $8-21 \mathrm{j}$ ) et la catégorie des absences très longues pour les femmes avec une intensité de l'effet très importante $(\beta=1.066, p<.01$ pour les absences de plus de $22 \mathrm{j})$.

La relation entre les ressources de l'emploi et l'absentéisme diffère pour les hommes et les femmes. Pour les hommes, la présence de moyens informationnels est associée à une diminution de la probabilité de s'absenter pour des durées longues. A l'inverse, pour les hommes, la présence de moyens humains est associée à une augmentation de la probabilité de s'absenter pour des durées très

\footnotetext{
${ }^{8}$ Hosmer et Lemeshow (2000, p.167) notent que «ces valeurs faibles de $R^{2}$ dans la régression logistique posent problème lorsqu'elles sont exposées à des lecteurs habitués aux $R^{2}$ de la régression linéaire ». Ils recommandent donc de ne pas les publier. Nous n'avons pas suivi les recommandations de Hosmer et Lemeshow (2000), de façon à laisser au lecteur la possibilité de comparer les pseudo- $\mathrm{R}^{2}$ de nos différents modèles de régression.

${ }^{9}$ C'est-à-dire à âge, ancienneté, statut d'empoi, type de temps de travail, catégorie socio-professionnel, taille de l'organisation, et la taille de l'organisation.

${ }^{10}$ C'est-à-dire à âge, ancienneté, profession exercée, statut d'empoi, type de temps de travail, catégorie socioprofessionnel, secteur d'acitivté et à taille de l'organisation identiques.
} 
longues. Pour les femmes, la présence de moyens cognitifs est associée à une diminution de la probabilité de s'absenter pour des durées courtes et très longues. Et la présence de moyens humains est associée à une probabilité moins forte de s'absenter pour des durées très longues, la présence de moyens informationnels étant elle associée à une probabilité plus faible de s'absenter pour des durées courtes. Lorsque l'analyse est menée sur l'ensemble de l'échantillon (tableau 5), seuls les moyens cognitifs ont un effet sur l'absentéisme.

Parmi les variables liées à l'organisation du travail, deux résultats intéressants sont à mettre en exergue. Les horaires individualisés sont associés à une diminution de la probabilité de s'absenter pour des durées courtes et longues pour les hommes mais, pour les femmes, l'effet des horaires flexibles sur la probabilité de s'absenter est non-significatif. Par ailleurs, la demande psychologique est associée à une augmentation de la probabilité de s'absenter pour des durées très longues pour les femmes. Mais l'effet de la demande psychologique sur l'absentéisme des hommes est non-significatif.

Les effets des variables liées aux relations sociales (soutien du supérieur, soutien des collègues, contrôle hiérarchique) sur l'absentéisme sont significatifs mais leurs amplitudes sont faibles, ce quelque soit le sexe.

$\underline{\text { Tableau } 4}$ : Régression multinomiale univariée sur l'échantillon total

\begin{tabular}{|c|c|c|c|}
\hline & $1-7 \mathbf{j}^{1}$ & $8-21 j^{1}$ & $>21 j^{1}$ \\
\hline \multicolumn{4}{|l|}{ Sexe } \\
\hline Hommes & $-.334 * * *$ & $-.425 * * *$ & $-.472 * * *$ \\
\hline Femmes & Ref & Ref & Ref \\
\hline -2 Logarithme de la vraisemblance & & 42335,57 & \\
\hline AIC & & 42491,57 & \\
\hline $\mathrm{BIC}$ & & 43123,83 & \\
\hline $\mathrm{Kki}^{2}$ & & $1162,20 * * *$ & \\
\hline ddl & & 75 & \\
\hline Pseudo- $\mathrm{R}^{2}$ de Nagelkerke & & .055 & \\
\hline$n$ & & 24486 & \\
\hline
\end{tabular}

Tableau 5 : Régression multinomiale multivariée sur l'échantillon total

\begin{tabular}{|c|c|c|c|}
\hline & $1-7 \mathbf{j}$ & $8-21 j$ & $>\mathbf{2 1 j}$ \\
\hline \multicolumn{4}{|c|}{ Variables de contrôle $^{1}$} \\
\hline \multicolumn{4}{|l|}{ Sexe } \\
\hline Hommes & $-.300^{* * * *}\left(0,74^{2}\right)$ & $-.300 * * *(0,74)$ & $-.206 *(0,81)$ \\
\hline Femmes & Ref & Ref & Ref \\
\hline \multicolumn{4}{|c|}{ Catégorie socio-professionnelle (CSP) } \\
\hline Cadres, professions Supérieures & ns & $-.474 * * *(0,46)$ & $-.514 * *$ \\
\hline Professions intermédiaires & ns & $-.211 *(0,65)$ & $-.283 *$ \\
\hline Employés & ns & ns & ns \\
\hline Ouvriers & Ref & Ref & $\operatorname{Ref}$ \\
\hline Ancienneté & $\mathrm{ns}$ & $\mathrm{ns}$ & ns \\
\hline Age & $-.030 * *(0,97)$ & $-.008 * *(0,99)$ & $.012 * * *(1,0)$ \\
\hline
\end{tabular}




\begin{tabular}{|c|c|c|c|}
\hline \multicolumn{4}{|l|}{ Fonction exercée } \\
\hline Installation, entretien, réparation & $.539 * *\left(1,7^{3}\right)$ & $.809 * *(5,1)$ & ns \\
\hline Gestion, comptabilité, administration & $.439 *(1,5)$ & $\mathrm{ns}$ & ns \\
\hline Recherche, étude, méthode, informatique & $.717 * * *(2,0)$ & ns & ns \\
\hline Direction générale & Ref & $\operatorname{Ref}$ & $\operatorname{Ref}$ \\
\hline \multicolumn{4}{|l|}{ Statut de l'emploi } \\
\hline Apprenti & $.628 * * *(1,9)$ & $.510 * *(1,6)$ & $.802 * *$ \\
\hline Stagiaire ou contrat aidé & $\mathrm{ns}$ & ns & ns \\
\hline Intérimaire & $-.538 *(0,08)$ & $-.683 *(0,5)$ & ns \\
\hline Contrat à durée déterminée (CDD) & $\mathrm{ns}$ & $-355 *(0,09)$ & ns \\
\hline Contrat à durée indéterminée (CDI) & $\mathrm{ns}$ & Ns & ns \\
\hline Fonctionnaire & Ref & Ref & Ref \\
\hline \multicolumn{4}{|l|}{ Secteur d'activité } \\
\hline Agriculture & $.141 * *(0,04)$ & ns & ns \\
\hline Industrie & $\mathrm{ns}$ & ns & ns \\
\hline Construction & Ref & Ref & Ref \\
\hline Taille de l'établissement & ns & $.000 * *(1,0)$ & $.000 *(1,0)$ \\
\hline \multicolumn{4}{|c|}{ Variables d'organisation du travail } \\
\hline Durée du travail & $0,009 * *(0,99)$ & $-.010 * *(0,99)$ & $-.017 * * *(0,98)$ \\
\hline Horaires individualisés & $\mathrm{ns}$ & $-.128 * * *(0,88)$ & $\mathrm{ns}$ \\
\hline Demande psychologique & ns & $\mathrm{ns}$ & ns \\
\hline $\begin{array}{l}\text { Utilisation et développement de } \\
\text { compétences }\end{array}$ & ns & $-.050 * *(0,95)$ & ns \\
\hline Autonomie décisionnelle & $-.062 * * *(0,94)$ & $-.047 * *(0,02)$ & $-.096 * * *(0,90)$ \\
\hline \multicolumn{4}{|c|}{ Variables de conditions de travail } \\
\hline Tensions avec le public & $\mathrm{ns}$ & $.081 *(1,1)$ & ns \\
\hline Agressions du public & $.254 * * *(1,3)$ & $.255 * * *(1,3)$ & $.294 * * *(1,34)$ \\
\hline Harcèlement moral & $.119 *(1,12)$ & $.220 * * *(1.2)$ & $323 * * *(1,38)$ \\
\hline Postures douloureuses & $-.119 * *(0,88)$ & $\mathrm{ns}$ & ns. \\
\hline Nuisances thermiques & $\mathrm{ns}$ & ns & ns \\
\hline Nuisances sonores & ns & $.270 * *(1,3)$ & ns \\
\hline Tensions avec le public & $\mathrm{ns}$ & $\mathrm{ns}$ & ns \\
\hline \multicolumn{4}{|c|}{ Variables de ressources de l'emploi } \\
\hline Moyens humains & $\mathrm{ns}$ & ns & ns \\
\hline Moyens informationnels & ns & ns & ns \\
\hline Moyens cognitifs & $-0,196 * * *(0,82)$ & $-.171 * * *(0,84)$ & $-0,235 * *(0,79)$ \\
\hline \multicolumn{4}{|c|}{ Variables de relations sociales } \\
\hline Soutien du supérieur & $-.25 * *$ & ns & ns \\
\hline Soutien des collègues & ns & ns & ns \\
\hline Contrôle hiérarchique & $.44 * * *$ & ns & ns \\
\hline-2 Logarithme de la vraisemblance & & 24725,27 & \\
\hline AIC & & 24983,27 & \\
\hline $\mathrm{BIC}$ & & 25961,42 & \\
\hline $\mathrm{Kki}^{2}$ & & $1158,11 * * *$ & \\
\hline ddl & & 126 & \\
\hline Pseudo- $\mathrm{R}^{2}$ de Nagelkerke & & .092 & \\
\hline$n$ & & 24486 & \\
\hline
\end{tabular}

${ }^{1}$ Seules les modalités ayant un coefficient significatif ont été présentées parmi les variables de contrôle. Les odds ratios sont indiqués entre parenthèses.

2 Dans un modèle Logit multinomial, l'odd-ratio reflète l'écart de probabilité d'une absence de $1-7 j, 8-21 j,+22 j$ par rapport à une modalité de référence ( 0 jour d'absence »). Si le odd-ratio est supérieur à 1 , le risque est plus élevé que pour la modalité de référence et, inversement, s'il est inférieur à 1 . Ainsi, toutes choses étant égales par ailleurs, les hommes ont $(1-0,74)=26 \%$ chances en moins que les femmes (modalité de référence) de s'absenter pour des durées comprises entre 1 et 7 jours.

3 Toutes choses étant égales par ailleurs, les salariés exerçant une fonction d'installation, d'entretien ou de réparation ont $70 \%$ plus de chances de s'absenter que les salariés exerçant une fonction de direction générale. 
Tableau 6 : Régression multinomiale multivariée sur le sous-échantillon des hommes

\begin{tabular}{|c|c|c|c|}
\hline & $\mathbf{1 - 7} \mathbf{j}$ & $8-21 j$ & $>\mathbf{2 1 j}$ \\
\hline \multicolumn{4}{|c|}{ Variables de contrôle } \\
\hline \multicolumn{4}{|l|}{ Sexe } \\
\hline Hommes & - & - & - \\
\hline Femmes & $\operatorname{Ref}$ & Ref & Ref \\
\hline \multicolumn{4}{|l|}{ Catégorie socio-professionnelle (CSP) } \\
\hline Cadres, professions Supérieures & ns & ns & $-.508 * *(0,60)$ \\
\hline Professions intermédiaires & ns & ns & $-.456^{* *}(0,63)$ \\
\hline Employés & ns & ns & ns \\
\hline Ouvriers & Ref & Ref & Ref \\
\hline Ancienneté & ns & ns & $\mathrm{ns}$ \\
\hline Age & $-.033 * * *(0,97)$ & ns & $.014 * *(0,49)$ \\
\hline \multicolumn{4}{|l|}{ Fonction exercée } \\
\hline Installation, entretien, réparation & ns & $1.012 *(2,7)$ & ns \\
\hline Gestion, comptabilité, administration & ns & ns & ns \\
\hline Recherche, étude, méthode, informatique & $.584 *(1,8)$ & ns & ns \\
\hline Nettoyage, gardiennage, travail ménager & $-.950 * *(0,33)$ & ns & ns \\
\hline Guichet, saisie, standard, secrétariat & $\mathrm{ns}$ & $.971 *(2,6)$ & ns \\
\hline Commerce, vente, technico-commercial & ns & $.899 *(2,4)$ & ns \\
\hline Direction générale & Ref & Ref & Ref \\
\hline \multicolumn{4}{|l|}{ Statut de l'emploi } \\
\hline Apprenti & ns & $.510 * *(1,4)$ & $1.125 * *(3,0)$ \\
\hline Stagiaire ou contrat aidé & ns & ns & ns \\
\hline Intérimaire & $-1.155 * * *(0,31)$ & $-.898 *(0,40)$ & ns \\
\hline Contrat à durée déterminée (CDD) & ns & $-1.054 *(0,35)$ & ns \\
\hline Contrat à durée indéterminée (CDI) & ns & $-.439 * *(0,64)$ & $\mathrm{ns}$ \\
\hline Fonctionnaire & Ref & Ref & Ref \\
\hline \multicolumn{4}{|l|}{ Secteur d'activité } \\
\hline Agriculture & ns & ns & $\mathrm{ns}$ \\
\hline Industrie & $.234 * *(1,3)$ & ns & ns \\
\hline Construction & $\mathrm{ns}$ & ns & ns \\
\hline Service & Ref & Ref & $\operatorname{Ref}$ \\
\hline Taille de l'établissement & $\mathrm{ns}$ & $.000 * *(1,0)$ & ns \\
\hline \multicolumn{4}{|c|}{ Variables d'organisation du travail } \\
\hline Durée du travail & $-.015 * *(0,004)$ & $-.020 * *(0,98)$ & $-.014 *(0,98)$ \\
\hline Horaires individualisés & $-.110 * *(0,30)$ & $-.225 * * *(0,79)$ & $\mathrm{ns}$ \\
\hline Demande psychologique & $\mathrm{ns}$ & ns & ns \\
\hline $\begin{array}{l}\text { Utilisation et développement de } \\
\text { compétences }\end{array}$ & ns & $-.064 * * *(0,93)$ & ns \\
\hline Autonomie décisionnelle & $-.049 *(0,95)$ & $-.055 *(0,95)$ & $-.098 * *(0,90)$ \\
\hline \multicolumn{4}{|c|}{ Variables de conditions de travail } \\
\hline Tensions avec le public & $\mathrm{ns}$ & $.153 * *(1,1)$ & ns \\
\hline Agressions du public & $.166^{*}(1,18)$ & $.198 *(1,2)$ & $\mathrm{ns}$ \\
\hline Harcèlement moral & ns & $\mathrm{ns}$ & $.308 * *(1,3)$ \\
\hline Postures douloureuses & ns & ns & $\mathrm{ns}$ \\
\hline Nuisances thermiques & ns & $.240 *(1,27)$ & ns \\
\hline Nuisances sonores & ns & $\mathrm{ns}$ & ns \\
\hline Tensions avec le public & ns & ns & ns \\
\hline \multicolumn{4}{|c|}{ Variables de ressources de l'emploi } \\
\hline Moyens humains & ns & ns & ns \\
\hline Moyens informationnels & ns & $-267 * *(0,76)$ & ns \\
\hline Moyens cognitifs & $\mathrm{ns}$ & $-.216 * *(0,80)$ & ns \\
\hline \multicolumn{4}{|c|}{ Variables de relations sociales } \\
\hline Soutien du supérieur & $-.048 * * *(0,95)$ & $-.053 * * *(0,94)$ & $-.059 * * *(0,94)$ \\
\hline Soutien des collègues & ns & ns & ns \\
\hline Contrôle hiérarchique & $.053 * * *(1,0)$ & $.057 * * *(1,0)$ & ns \\
\hline-2 Logarithme de la vraisemblance & & 11594,38 & \\
\hline AIC & & 11846,38 & \\
\hline $\mathrm{BIC}$ & & 12718,77 & \\
\hline $\mathrm{Kki}^{2}$ & & $610,68 * * *$ & \\
\hline $\mathrm{ddl}$ & & 123 & \\
\hline Pseudo- $\mathrm{R}^{2}$ de Nagelkerke & & .097 & \\
\hline$n$ & & 24486 & \\
\hline
\end{tabular}


${ }^{1}$ Seules les modalités ayant un coefficient significatif ont été présentées parmi les variables de contrôle. Les Odds Ratios sont indiqués entre parenthèses.

Tableau 7 : Régression multinomiale multivariée sur le sous-échantillon des femmes

\begin{tabular}{|c|c|c|c|}
\hline & $1-7 \mathbf{j}$ & $8-21 j$ & $>\mathbf{2 1 j}$ \\
\hline \multicolumn{4}{|c|}{ Variables de contrôle } \\
\hline \multicolumn{4}{|l|}{ Sexe } \\
\hline Hommes & - & - & - \\
\hline Femmes & Ref & Ref & Ref \\
\hline \multicolumn{4}{|l|}{ Catégorie socio-professionnelle (CSP) } \\
\hline Cadres, professions Supérieures & ns & ns & ns \\
\hline Professions intermédiaires & ns & ns & ns \\
\hline Employés & ns & $\mathrm{ns}$ & $\mathrm{ns}$ \\
\hline Ouvriers & Ref & Ref & Ref \\
\hline Ancienneté & ns & ns & ns \\
\hline Age & $-.035 * * *(.96)$ & $-.014 * * *(.98)$ & ns \\
\hline \multicolumn{4}{|l|}{ Fonction exercée } \\
\hline Installation, entretien, réparation & ns & ns & ns \\
\hline Gestion, comptabilité, administration & $.838 *(2.3)$ & ns & ns \\
\hline Recherche, étude, méthode, informatique & $.782 *(2.1)$ & ns & ns \\
\hline Commerce, vente, technico-commercial & $.838 *(2.3)$ & ns & ns \\
\hline $\begin{array}{l}\text { Enseignement, santé, information et autre } \\
\text { cas }\end{array}$ & $.796 *(2.2)$ & ns & ns \\
\hline Direction générale & Ref & Ref & Ref \\
\hline \multicolumn{4}{|l|}{ Statut de l'emploi } \\
\hline Apprenti & $.718 * * *(2.0)$ & ns & ns \\
\hline Stagiaire ou contrat aidé & $\mathrm{ns}$ & ns & ns \\
\hline Intérimaire & ns & ns & ns \\
\hline Contrat à durée déterminée (CDD) & ns & ns & $\mathrm{ns}$ \\
\hline Contrat à durée indéterminée (CDI) & ns & ns & $.562 * * *(1.75)$ \\
\hline Fonctionnaire & Ref & Ref & Ref \\
\hline \multicolumn{4}{|l|}{ Secteur d'activité } \\
\hline Industrie & ns & ns & ns \\
\hline Construction & ns & $\mathrm{ns}$ & ns \\
\hline Service & Ref & Ref & Ref \\
\hline Taille de l'établissement & $.000 * * *(1.0)$ & $.000 * * *(1.0)$ & $.000 *(1.0)$ \\
\hline \multicolumn{4}{|c|}{ Variables d'organisation du travail } \\
\hline Durée du travail & $\mathrm{ns}$ & ns & $-.018 * *(.98)$ \\
\hline Horaires individualisés & ns & ns & $\mathrm{ns}$ \\
\hline Demande psychologique & ns & ns & $.024 *(1.0)$ \\
\hline $\begin{array}{l}\text { Utilisation et développement de } \\
\text { compétences }\end{array}$ & ns & $-.041 * *(.95)$ & ns \\
\hline Autonomie décisionnelle & $-.075 * * *$ & ns & $-.092 * *(.91)$ \\
\hline \multicolumn{4}{|c|}{ Variables de conditions de travail } \\
\hline Tensions avec le public & ns & $\mathrm{ns}$ & ns \\
\hline Agressions du public & $.314 * * *(1.4)$ & $.272 * * *(1.13)$ & $.355 * * *(1.4)$ \\
\hline Harcèlement moral & $.151 *(1,1)$ & $.250 *(1.28)$ & $.365 * * *(1.4)$ \\
\hline Postures douloureuses & $-.129 *(0,87)$ & $\mathrm{ns}$ & $\mathrm{ns}$ \\
\hline Nuisances thermiques & ns & ns & $1.066 * * *(2.9)$ \\
\hline Nuisances sonores & ns & ns & ns \\
\hline Tensions avec le public & ns & ns & ns \\
\hline \multicolumn{4}{|c|}{ Variables de ressources de l'emploi } \\
\hline Moyens humains & $\mathrm{ns}$ & ns & $-.206 *(.81)$ \\
\hline Moyens informationnels & $.176^{* *}(1.19)$ & ns & ns \\
\hline Moyens cognitifs &,$- 247 * * *(.78)$ & ns &,$- 263 * *(.79)$ \\
\hline \multicolumn{4}{|c|}{ Variables de relations sociales } \\
\hline Soutien du supérieur & $\mathrm{ns}$ & $-.046 * *(.95)$ & $-.057 * *(.94)$ \\
\hline Soutien des collègues & ns & ns & ns \\
\hline Contrôle hiérarchique & $.035^{* *}(1.0)$ & ns & ns \\
\hline -2 Logarithme de la vraisemblance & & 12960,06 & \\
\hline $\mathrm{AIC}$ & & 13212,06 & \\
\hline $\mathrm{BIC}$ & & 14075,64 & \\
\hline
\end{tabular}




\begin{tabular}{|c|c|}
\hline $\mathrm{Khi}^{2}$ & $600,70 * * *$ \\
\hline ddl & 123 \\
\hline Pseudo-R ${ }^{2}$ de Nagelkerke & .096 \\
\hline$n$ & 24486 \\
\hline
\end{tabular}

\section{Discussion}

La contribution principale de notre étude est l'effet «écran de fumée » de la variable «sexe »sur l'absentéisme : le sexe en tant que variable explicative en soi constitue un substitut aux conditions de travail qui lui sont associées et qui différent selon que l'on est un homme ou une femme (Deguire et Messing, 1995), confirmant plusieurs études québécoises menées par Messing et alii (Messing et alii, 1998 : Deguire et Messing, 1995 ; Messing et alii, 2011).

Plus précisément, nos résultats montrent que des analyses menées sur un échantillon combinant hommes et femmes entraînent une perte d'information comparativement à des analyses menées séparément sur des sous-échantilllons composés soit exclusivement de femmes, soit exclusivement d'hommes. Lorsque les femmes et les hommes sont inclus dans l'échantillon et que le sexe est considéré comme une variable indépendante, plusieurs relations entre déterminants organisationnels et absentéisme ne sont plus significatives. Ceci peut s'expliquer par le fait que de nombreuses relations entre déterminants organisationnels et absentéisme, significatives pour les salariés d'un sexe donné, sont confondues ou fortement atténuées lorsque l'on inclut les salariés de l'autre sexe (Messing et alii, 1998). Ainsi, la demande psychologique n'a aucun effet significatif sur chacune des catégories d'absence lorsque l'analyse est menée sur un échantillon mixte. Toutefois, lorsque les analyses sont menées séparément, la demande psychologique est un antécédent important des absences de longue durée pour les femmes. Or ce résultat ne se retrouve pas pour les hommes quelque soit la catégorie d'absence considérée. Ces derniers résultats confirment ceux de plusieurs études (Niedhammer et alii, 2006; Melchior et alii, 2003) mais infirment les résultats de North et alii (1996) qui ne montrent aucune différence sexuée dans la relation entre la demande psychologique et les absences-maladie. De même, les horaires individualisés ont un effet significatif sur l'absentéisme des hommes - ils en diminuent la probabilité de s'absenter - mais non sur celui des femmes. Ce dernier résultat montre que les horaires individualisés, souvent présentés comme un moyen d'améliorer la conciliation pour les femmes entre leur vie privée et leur vie professionnelle ${ }^{11}$, ne suffisent pas à diminuer leur probabilité de s'absenter, toutes choses étant égales par ailleurs. Nous pouvons supposer que les femmes bénéficiant d'horaires flexibles sont aussi celles qui sont confrontées à des conditions de travail plus pénibles, les horaires flexibles agissant alors comme une arme à double-tranchant (Schieman et alii, 2009).

\footnotetext{
${ }^{11}$ Voir, par exemple, De nouvelles organisations du travail conciliant égalité femme/homme et performance des enreprises. Perspectives internationale, Actes de séminaire, Centre d'analyse stratégique (CAS), 4 novembre 2011.
} 
Par ailleurs, nos résultats montrent que, comparativement à une analyse multivariée, l'amplitude la relation entre la variable sexe et les absences courtes et longues diminuent fortement (respectivement, $\beta=-.334, p<.01->\beta=-.300, p<.01 ; \beta=-.425, p<.01->\beta=-.300, p<.01$, tableaux 4 et 5) et encore plus fortement pour les absences de longue durée pour lesquelles la relation est significative seulement au seuil d'erreur de $10 \%(\beta=-.472, p<.01 \rightarrow \beta=-.206, p<.1$, tableaux 4 et 5$)$. Ces derniers résultats sont convergents avec ceux de Messing et alii (1998) et confirment la thèse selon laquelle ce sont principalement une organisation et des conditions de travail sexuées qui expliquent les différences d'absentéisme entre les hommes et les femmes. Les tris croisés (tirés de l'enquête Sumer 2003) présentés dans les tableaux 8 et $9^{12}$ confirment une organisation et des conditions de travail sexuées ainsi qu'une répartition sexuée des ressources de l'emploi. Ainsi les femmes, cadres ou ouvrières ont significativement $\left(\mathrm{Khi}^{2}, p<.05\right)$ moins d'autonomie décisionnelle dans leur travail. De même, elles utilisent et développent significativement $\left(\mathrm{Khi}^{2}, p<.05\right)$ moins leurs compétences dans leur travail. Les femmes cadres sont significativement $\left(\mathrm{Khi}^{2}, p<.05\right)$ plus exposées que les hommes cadres aux agressions, aux tensions avec le public. De plus, les femmes ouvrières sont significativement $\left(\mathrm{Khi}^{2}, p<.05\right)$ plus exposées que les hommes aux postures douloureuses. Enfin, la répartition des ressources de l'emploi (moyens informationnels, cognitifs et humains) est significativement $\left(\mathrm{Khi}^{2}, p<.05\right)$ réalisée au désavantage des femmes cadres. Plus significativement $\left(\mathrm{Khi}^{2}, p<.05\right)$ que les hommes, les femmes manquent d'informations, de formations ainsi que de moyens humains suffisants pour effectuer correctement leur travail.

Tableau 8: Une organisation du travail et des conditions de travail sexuées (en pourcentage)

\begin{tabular}{|c|c|c|c|c|}
\hline \multirow[b]{3}{*}{$\begin{array}{l}\text { Autonomie } \\
\text { décisionnelle } \\
\text { Homme }\end{array}$} & \multicolumn{2}{|c|}{ Ouvrier } & \multicolumn{2}{|c|}{ Cadre } \\
\hline & Faible & Forte & Faible & Forte \\
\hline & 68,8 & 31,2 & 35,5 & 64,5 \\
\hline Femme & 81,4 & $18,6^{1}$ & 39,3 & $60,7^{1}$ \\
\hline Ensemble & 71,0 & 29,0 & 36,6 & 63,4 \\
\hline $\begin{array}{l}\text { Utilisation et } \\
\text { développement de } \\
\text { compétences }\end{array}$ & Faible & Forte & Faible & Forte \\
\hline Homme & 58,3 & 41,7 & 18,4 & 81,6 \\
\hline Femme & 81,3 & $18,7^{1}$ & 25,2 & $74,8^{1}$ \\
\hline Ensemble & 62,3 & 37,7 & 20,3 & 79,7 \\
\hline $\begin{array}{l}\text { Tension avec le } \\
\text { public }\end{array}$ & Faible & Forte & Faible & Forte \\
\hline Homme & 59,7 & 40,3 & 83,7 & 16,3 \\
\hline Femme & 52,2 & $47,8^{1}$ & 77,1 & $22,9^{1}$ \\
\hline Ensemble & 58,8 & 41,2 & 81,8 & 18,2 \\
\hline $\begin{array}{l}\text { Postures } \\
\text { douloureuses }\end{array}$ & & & & \\
\hline Homme & 45,8 & 54,2 & 81,4 & 18,6 \\
\hline Femme & 40,5 & $59,5^{1}$ & 80,2 & 19,8 \\
\hline
\end{tabular}
${ }^{12}$ Les variables présentées dans les tableaux 8 et 9 sont dichotomisées à la médiane pour l'ensemble de la
population, fort : valeur supérieure à la médiane, faible : valeur inférieure à la médiane) 


\begin{tabular}{lcccc}
\hline Ensemble & 44,9 & 55,1 & 81,0 & 19,0 \\
\hline Agressions & & & & 12,5 \\
Homme & 90,8 & $9,2^{1}$ & 87,5 & $18,0^{1}$ \\
Femme & 93,4 & 6,6 & 82,0 & 14,1 \\
\hline Ensemble & 91,3 & 8,7 & 85,9 & 14,1 \\
\hline Harcèlement & & & & 18,7 \\
Homme & 83,0 & 17,0 & 85,9 & 15,4 \\
\hline Femme & 83,3 & $16,7^{1}$ & 81,3 & 84,6 \\
\hline
\end{tabular}

${ }^{1}:$ Test du $\mathrm{Khi}^{2}$ significatif $(p<0,05)$

$\underline{\text { Tableau } 9}$ : Une répartition sexuée des ressources de l'emploi (en pourcentage)

\begin{tabular}{lcccc}
\hline & \multicolumn{2}{c}{ Ouvrier } & & Cadre \\
\cline { 2 - 4 } Moyens & Faible & Forte & Faible & Forte \\
\cline { 2 - 5 } informationnels & & & & 79,5 \\
Homme & 15,7 & 84,3 & 20,5 & $72,8^{1}$ \\
Femme & 17,3 & 82,7 & 27,2 & 7,6 \\
\hline Ensemble & 16,0 & 84,0 & 22,4 & 71,9 \\
Moyens humains & & & & $65,3^{1}$ \\
Homme & 22,5 & 77,5 & 28,1 & 70,0 \\
Femme & 23,2 & 76,8 & 34,7 & 86,4 \\
\hline Ensemble & 22,7 & 77,3 & 30,0 & $83,5^{1}$ \\
\hline Moyens cognitifs & & & & 85,5 \\
Homme & 19,0 & 81,0 & 13,6 & 16,5 \\
Femme & 21,0 & $79,0^{1}$ & 14,5 & \\
\hline Ensemble & 19,3 & 80,7 & & \\
\hline
\end{tabular}

${ }^{1}:$ Test du $\mathrm{Khi}^{2}$ significatif $(\mathrm{p}<0,05)$

Notre étude comporte toutefois plusieurs limites. Tout d'abord, toutes les variables ont été évaluées en coupe instantanée, ce qui signifie que nous ne pouvons pas tirer de conclusions fermes sur les inférences causales entre ces variables. Seule une étude longitudinale pourrait donner des conclusions fermes sur le sens de la causalité. Ensuite, l'étude étant en coupe transversale, nous n'avons analysé que les effets à court terme des facteurs organisationnels sur le harcèlement moral. Par ailleurs, notre étude n'inclut pas l'étude des effets sur l'absentéisme de pratiques de gestion des ressources humaines différenciées selon le sexe des salariés.

Pour conclure, notre étude nous a permis de vérifier et de montrer l'importance des déterminants organisationnels pour expliquer les différences de taux d'absentéisme entre les hommes et les femmes. Pour autant, le choix de la mesure de l'absentéisme opéré et la distinction entre très longue, longue et courte absences qui en résulte ne nous permettent pas d'expliquer la différence des comportements d'absence-maladie suivant le sexe du salarié. Une mesure combinant durée et fréquence permet par contre d'élaborer une typologie des absences-maladie (Bouville, 2009) et pourrait s'avérer plus heuristique pour comprendre les comportements différenciés des deux sexes. 
Notre étude aboutit à un résultat inattendu: elle met en exergue une discrimination hommes/femmes très peu explorée: celle du fonctionnement interne de l'organisation caractérisé par des modes de division et de coordination du travail différents selon le sexe du salarié.

La frontière en fonction du sexe du salarié, du point de vue de l'organisation du travail, des conditions de travail, des relations sociales et des ressources de l'emploi, a été explorée par plusieurs travaux de recherche développés au sein des Gender Studies - par exemple, dans les travaux pionniers de Joan Acker (1990) - mais reste très méconnue en sciences de gestion. Tout juste sont reconnues les discriminations en matière de carrière professionnelle (le plafond de verre) et de rémunération. Ces discriminations sont parfois cependant contestées. Ainsi le différentiel de rémunération entre les hommes et les femmes s'expliquerait par un différentiel de qualification (ou, encore, moins mesurable par un différentiel de compétence). Toutefois, notre étude met en lumière une question : ce différentiel entre salariés hommes et femmes n'est-il pas encore plus ancré dans le fonctionnement des organisations c'est-à-dire dans les caractéristiques organisationnelles de ces lieux de travail ?

Cette frontière est interne aux organisations qui fonctionnent avec une main d'œuvre mixte. Elle passe également entre les organisations employant une main d'œuvre essentiellement féminine et celles qui emploient une main d'œuvre essentiellement masculine. Un approfondissement de cette recherche consisterait à mener des études de cas dans des organisations à main d'œuvre mixte. L'analyse des caractéristiques organisationnelles sexuées (la répartition de la main d'œuvre, selon le sexe, au sein des équipes de travail, la reconnaissance ou non de certains savoirs et savoir-faire dans les qualifications et rémunérations octroyées aux hommes et aux femmes ...) permettrait de mieux comprendre comment est construite cette frontière entre les sexes au sein des organisations. Une autre piste possible d'exploration consisterait à opérer des comparaisons entre entreprises à main d'œuvre essentiellement féminine et celles à main d'œuvre essentiellement masculine, à processus de production très proches.

\section{Bibliographie}

Acker, J. (1990), Hierarchies, Jobs, Bodies: A Theory of Gendered Organization, Gender and Society, vol. 4, n 2 , p. 139-158.

Alis, D., Dumas, M. (2003), 35 heures, soutien organisationnel perçu et harmonisation vie familiale / vie professionnelle, Revue de Gestion des Ressources Humaines, vol. 50, p. 37-56.

Baba, V.V. (1990), Methodological Issues in Modeling Absence: A Comparison of Least Squares and Tobit Analyses, Journal of Applied Psychology, vol. 75, n 4, p. 428-432.

Bouville, G. (2009), L'influence de l'organisation et des conditions de travail sur l'absentéisme. Analyse quantitative et étude de cas. Thèse de Doctorat en Sciences de Gestion, Université de Rennes 1 .

Bratberg, E., Dahl, S.A., Risa, A.E. (2002), The "double burden". Do combinations of career and family obligations increase sickness absence among women?, European Sociological Review, vol.18, n², p. 233-249. 
Brugueilles C., Sebille, P. (2009), La participation des pères aux soins et à l'éducation des enfants. L'influence des rapports sociaux de sexe entre les parents et entre les générations », Politiques sociales et familiales, vol. 95, p. 19-32.

Chappert F. (2009), Genre et conditions de travail, Etudes et documents, ANACT.

Côté, D., Haccoun R.R. (1991), L'absentéisme des femmes et des hommes : Une méta-analyse, Canadian Journal of Administrative Sciences, vol. 8, $\mathrm{n}^{\circ}$ 2, p. 130-139.

Coutrot, T., Wolff, L. (2005), L'impact des conditions de travail sur la santé : une expérience méthodologique, Rapport de recherche du CEE, vol. 23.

Deguire, S., Messing, M. (1995), L'étude de l'absence au travail a-t-elle un sexe?, Recherches féministes, vol. 8, n² 2, p. 9-30.

De Jonge, J., Reuvers, M.M., Houtman, I.L., Bongers, P.M., Kompier, M.A. (2000), Linear and nonlinear relations between psychosocial job characteristics, subjective outcomes, and sickness absence: baseline results from SMASH. Study on Musculoskeletal Disorders, Absenteeism, Stress, and Health, Journal of Occupational Health Psychology, vol. 5, n 2, p. 256-268.

Dumas, M. (2008), Vie personnelle et vie professionnelle. Vers un nouvel équilibre dans l'entreprise ?, Cormelles-Le-Royal, EMS.

Erickson, R.J., Nichols, L., Ritter, C. (2000), Family influences on absenteeism: Testing an expanded process model, Journal of Vocational Behavior, vol. 57, n² 2, p. 246-272.

Farell, D., Stamm, C.L. (1988), Meta-analysis of the correlates of employee absence, Human Relations, vol. 41, n 3 , p. 211-227.

Ferrie, J.E., Kivimäki, M., Head, J., Shipley, M.J., Vahtera, J., Marmot, M.G. (2005), A comparison of self-reported sickness absence with absences recorded in employers' registers: evidence from the Whitehall II study, Occupational and Environmental Medicine, vol. 62, n 2, p. 74-79.

Goff, S.J., Mount, M.K, Jamison, R.L (1990), Employee supported child care, work/famlily conflict, and absenteeism: a field study, Personnel Psychology, vol. 43, n 4, p. 793-809.

Greene, W.H. (2003), Econometric Analysis, New Jersey, Prentice Hall.

Harrison, D.A., Martocchio, J.J. (1998), Time for absenteeism: a 20-year review of origins, offshoots and outcomes, Journal of management, vol. 24, $\mathrm{n}^{\circ} 3$, p. 305-330.

Hensing, G., Alexanderson, K. (2004), The association between sex segregation, working conditions, and sickness absence among employed women. Occupational and Environmental Medicine, vol. 61, $\mathrm{n}^{\circ}$ 2, p. 1-6.

Hosmer, D.W., Lemeshow, S. (2000), Applied Logistic Regression (2nd Edition), New York, Wiley \& Sons.

IRES (2005), Les mutations de l'emploi en France, Paris, La Découverte.

Ichino, A., Moretti, E. (2009), Biological Gender Differences, Absenteeism, and the Earnings Gap, American Economic Journal: Applied Economics, vol. 1, n 1, p. 183-218.

Karasek, R.A., Theorell, T. (1990), Healthy work, stress, productivity and the reconstruction of work life, New York, Basic Books.

Laaksonen, M., Martikainen, P., Rahkonen, O., Lahelma, E. (2008), Explanations for gender differences in sickness absence: from middle-aged municipal employees from Finland, Occupational and Environmental Medicine, vol. 65, $\mathrm{n}^{\circ}$ 5, p. 325-330.

Gollac, M., Volkoff, S. (2007), Les conditions de travail, Paris, La Découverte.

Leymann, H. (1996), The content and development of mobbing at work, European Journal of Work and Organizational Psychology, vol. 5, $\mathrm{n}^{\circ} 2$, p. 165-184. 
Marmot, M., Feeney, A., Shipley, M., North, F., Syme, S.L. (1995), Sickness absence as a measure of health status and functioning: from the UK Whitehall II study, Journal of Epidemiology and Community Health, vol. 49, n 2, p. 124-130.

Mastekaasa, A. (2000), Parenthood, gender and sickness absence, Social Science and Medicine, vol. $50, \mathrm{n}^{\circ} 12$, p. $1827-1842$.

Mastekaasa, A., Olsen, K.M. (1998), Gender, absenteeism, and job characteristics: A fixed effects approach, Work and Occupation, vol. 25, n 2 , p. 195-228.

Melchior, M., Niedhammer, I., Berkman, L.F., Goldberg, M. (2003), Do psychosocial work factors and social relations exert independent effects on sickness absence? A six year prospective study of the GAZEL cohort, Journal of Epidemiology and Community Health, vol. 57, $\mathrm{n}^{\circ}$ 4, p. 285-293.

Messing K, Tissot F, Saurel-Cubizolles MJ, Kaminski M, Bourgine M. (1998), Sex as a variable can be a surrogate for some working conditions: factors associated with sickness absence, Journal of Occupational and Environmental Medicine, vol. 40, n ${ }^{\circ}$ 3, p. 250-260.

Messing, K., Lippel, K., Stock, S., Tissot, F. (2011), Si le bruit rend sourd, rend-il nécessairement sourde? Le défi d'appliquer l'analyse différenciée selon le sexe à la recherché d'informations sur la santé et la sécurité du travail, Revue mutlidisciplinaire sur l'emploi, le syndicalisme et le travail, vol. $6, \mathrm{n}^{\circ} 2$, p. 3-25.

Mittlböck, M., Heinzl, H. (2001), A note on $\mathrm{R}^{2}$ measures for Poisson and logistic regression models when both models are applicable, Journal of Clinical Epidemiology, vol. 54, p. 99-103.

Niedhammer, I., Chastang, J.F, David. S. (2006), Importance of psychosocial work factors on general health outcomes in the national French SUMER survey, Occupational Medicine, vol. 58, ${ }^{\circ}$ 1, p. 1524.

North, F.M., Syme, S. L., Feeney, A., Shipley, M., Marmot, M. (1996), Psychosocial Work Environment and Sickness Absence among British Civil Servants: The Whitehall II Study, American Journal of Public Health, vol. 86, $\mathrm{n}^{\circ}$ 3, p. 332-340.

Pampel, F.C. (2000), Logistic regression. A primer, Thousand Oaks, Sage.

Patton, E., Johns, G. (2007), Women's absenteeism in the popular press: Evidence for a gendrespecific absence culture, Human Relations, vol. 60, n 11, p. 1579-1612.

Schieman S, Melissa M, and Paul G (2009), When Work Interferes with Life: Work-Nonwork Interference and the Influence of Work-Related Demands and Resources, American Sociological Review, vol. 74, n 6, p. 966-988.

Vaananen, A., Salla, T.T., Raija, K., Pertti, M., Jussi, V., José M.P (2003), Job characteristics, physical and psychological symptoms, and social support as antecedents of sickness absence among men and women in the private industrial sector, Social Science and Medicine, vol. 57, $\mathrm{n}^{\circ}$ 5, p. $807-$ 824.

Vistnes, J. P. (1997), Gender differences in days lost from work due to illness, Industrial \& Labor Relations Review, vol. 50, n 2, p. 304-323.

Watson, C. J. (1981), An evaluation of some aspects of the Steers and Rhodes model of employee attendance, Journal of Applied Psychology, vol. 66, n ${ }^{\circ}$, p. 385-389.

Vlassenko, E., Willard, J.C. (1984), Absentéisme : le poids des facteurs collectifs, Economie et Statistique, vol. 164, p. 39-51.

Voss, M., Floderus, B., Diderichsen, F. (2001), Physical, psychosocial, and organisational factors relative to sickness absence: a study based on Sweden post, Occupational and Environmental Medicine, vol. 58, $\mathrm{n}^{\circ}$ 3, p. 178-184.

Voss, M., Stark, S., Alfredsson, L., Vingard, E., Josephson, M. (2008), Comparisons of self-reported and register data on sickness absence among public employees in Sweden, Occupational and Environmental Medicine, vol. 65, $\mathrm{n}^{\circ}$ 1, p. 61-67. 
